\title{
Clinical implication of ocular torsion in peripheral vestibulopathy
}

\author{
Jin Woong Choi · Seong Il Kang • Ji Hye Rhee • \\ Byeong Yoon Choi $\cdot \mathrm{Ji}$-Soo Kim $\cdot$ Ja-Won Koo
}

Received: 16 March 2013 / Accepted: 11 February 2014 / Published online: 8 March 2014

(C) Springer-Verlag Berlin Heidelberg 2014

\begin{abstract}
Acute unilateral vestibular loss presents as ocular torsion (OT) and caloric unilateral weakness (UW). However, the amount of OT is frequently dissociated from UW depending on when the examination was performed and the extent and cause of the vestibular lesion. This study evaluated the relationship between OT and UW in peripheral vestibular diseases, including Ménière's disease (MD) and vestibular neuritis $(\mathrm{VN})$, and determined whether it contributed to OT as a means of differentiating between the two diseases. A retrospective chart review was performed in 64 patients with VN and 67 patients with MD. We divided the patients into three groups according to the interval from symptom onset to when the tests were performed: within 7 (group A), from 8 to 30 (group B) and over 30 (group C) days. UW, OT and the chronological correlation/dissociation between the two parameters were analyzed. For the 64 patients with VN, the degree of OT and severity of UW were positively correlated in group
\end{abstract}

J. W. Choi

Department of Otolaryngology-Head and Neck Surgery, Chungnam National University School of Medicine, Daejeon, South Korea

S. I. Kang · J. H. Rhee $\cdot$ B. Y. Choi · J.-W. Koo $(\bowtie)$ Department of Otorhinolaryngology, Seoul National University College of Medicine, Seoul National University Bundang Hospital, 166 Gumi-ro, Bundang-gu, Seongnam,

Gyeonggi-do 463-707, South Korea

e-mail: jwkoo99@snu.ac.kr

\section{B. Y. Choi · J.-W. Koo}

Research Center for Sensory Organs, Seoul National

University Medical Research Center, Seoul, South Korea

J.-S. Kim

Department of Neurology, Seoul National University

Bundang Hospital, Seongnam, South Korea
A $(r=0.749, P<0.001)$. OT and UW were significantly dissociated with time $(P<0.001)$. For the 67 patients with $\mathrm{MD}$, no correlation between the degree of OT and severity of UW was seen in MD group A. No significant dissociation change was revealed among the groups $(P=0.114)$. The OT abnormality is remarkable during the acute phase of $\mathrm{VN}$, whereas it might not be remarkable immediately after a vertigo attack in MD. This finding can be used to differentiate MD and VN, especially when no definite hearing loss is seen or VN recurs.

Keywords Ocular torsion - Peripheral vestibulopathy · Vestibular neuritis $\cdot$ Meniere disease $\cdot$ Dissociation

\section{Introduction}

A sudden loss of unilateral vestibular function leads to deficits in both the static state related to the tonic imbalance of the resting activity of the vestibular nuclei and the dynamic state associated with the response to motion. These vestibular system abnormalities can be demonstrated using various vestibular function tests. Fundus photography is used to evaluate the ocular torsion (OT) resulting from the static imbalance of the roll plane sensed by the otolith receptors in the utricles [1]. The OT abnormality in acute unilateral vestibular loss usually resolves within days or weeks $[2,3]$. Caloric testing examines the relative status of each horizontal semicircular canal separately and reflects the dynamic vestibular imbalance evoked by caloric stimulation. The disappearance of any caloric abnormality is slower than for the OT abnormality and can take several months $[2,4]$.

A time difference in the recovery of the static and dynamic imbalance has been demonstrated in vestibular neuritis $(\mathrm{VN})$, representing acute non-fluctuating vestibular 
Table 1 Demographics, caloric unilateral weakness and ocular torsion in patients with vestibular neuritis
$V N$ vestibular neuritis, $S D$ standard deviation, $I O D$ interocular difference

\begin{tabular}{lllll}
\hline & $\begin{array}{l}\text { Group A } \\
(n=28)\end{array}$ & $\begin{array}{l}\text { Group B } \\
(n=12)\end{array}$ & $\begin{array}{l}\text { Group C } \\
(n=24)\end{array}$ & $P$ value \\
\hline $\begin{array}{l}\text { Age, mean } \pm \text { SD (year) } \\
\text { Sex (M:F) }\end{array}$ & $\begin{array}{l}50.9 \pm 12.5 \\
14: 14\end{array}$ & $\begin{array}{l}48.6 \pm 17.3 \\
6: 6\end{array}$ & $\begin{array}{l}53.8 \pm 17.7 \\
16: 8\end{array}$ & 0.613 \\
Unilateral weakness & & & 0.429 \\
Mean \pm SD $(\%)$ & $69.2 \pm 19.3$ & $71.3 \pm 29.9$ & $72.7 \pm 33.0$ & 0.895 \\
Abnormal $(\%)$ & $28 / 28(100)$ & $10 / 12(83.3)$ & $19 / 24(79.2)$ & 0.016 \\
IOD & & & & \\
Mean \pm SD $\left({ }^{\circ}\right)$ & $13.2 \pm 9.5$ & $5.7 \pm 3.9$ & $4.6 \pm 3.1$ & I vs. II: 0.003 \\
& & & & I vs. III: $<0.001$ \\
Abnormal $(\%)$ & $17 / 28(60.7)$ & $2 / 12(16.7)$ & $2 / 24(8.3)$ & $\begin{array}{l}<0.001 \\
\text { Dissociation }(\%)\end{array}$ \\
\hline
\end{tabular}

loss $[2,5]$. After VN, the recovery of canal paresis varies from 50 to $86 \%$ within 2 years, while the recovery of asymmetric OT is $\sim 85 \%$ within 1 month [4, 6]. To our knowledge, however, no study has examined the time difference in the recovery of the static and dynamic imbalance in Ménière's disease (MD), although MD is a common peripheral vestibular disorder with an incidence of 4.3-15.3 per 100,000 [7]. In addition, it is not clear whether the OT abnormality indicating static imbalance reflects a recent vertigo attack during the follow-up of MD characterized by recurrent episodic vertigo.

Therefore, this study assessed the correlation between caloric unilateral weakness (UW) and OT according to the time course from the onset of dizziness in MD and in VN. We also examined whether the OT abnormality reflects a recent attack of vertigo in $\mathrm{MD}$.

\section{Materials and methods}

This study recruited patients diagnosed with VN or unilateral definite MD from July 2006 through December 2011 for whom audiovestibular function tests, including caloric tests and fundus photography, were available. VN was defined using the following criteria: (1) a history of acute-onset prolonged vertigo, (2) no hearing loss, (3) no additional neurological signs or symptoms and (4) UW on caloric test [8]. Definite MD was diagnosed using the guideline proposed by American Academy of Otolaryngology-Head and Neck Surgery [9].

Sixty-four patients with VN (mean age 51.5 (range 9-80) years; 36 males, 28 females) and 67 with MD (mean age 54.6 (range 25-77) years; 25 males, 42 females) were enrolled. The patients were classified into three groups according to the interval from the onset of the latest true vertigo attack lasting for more than 20 min to when the diagnostic tests were performed: group A, within 7 days; group $\mathrm{B}$, from 8 to 30 days; and group $\mathrm{C}$, more than 30 days.
OT indicating static vestibular imbalance was assessed using fundus photography with a scanning laser ophthalmoscope (Fundus camera CF-60 UVI, Canon, Tokyo, Japan), with the patient's head upright. OT was determined by measuring the angle between a horizontal line running through the center of the optic disc and a line connecting the center of the optic disc and fovea. Intorsion with a negative value of the angle, extorsion $>12.6^{\circ}$, or an interocular difference $\left(\right.$ IOD) $>8.8^{\circ}$ were considered as abnormal [4]. The bithermal caloric test of dynamic vestibular imbalance was evaluated by using binaural alternate irrigation for $30 \mathrm{~s}$ with $300 \mathrm{~mL}$ of cold $\left(30{ }^{\circ} \mathrm{C}\right)$ and warm $\left(44^{\circ} \mathrm{C}\right)$ water. UW was calculated using Jongkees' formula and a response difference $\geq 25 \%$ between the ears was defined as abnormal [10]. Dissociation was defined when the degree of OT was not in accordance with the severity of UW. The study protocol was approved by the Internal Review Board of Seoul National University Bundang hospital (IRB No. B-1210-176-112).

Statistical analysis using the SPSS ver. 18 software (SPSS, Chicago, IL) was performed to identify trends for the time course of abnormal OT and UW according to the groups (linear by linear association Chi-square test) and to calculate the correlations between OT and UW within each group (Pearson's correlation analysis). Analysis of variance (ANOVA) was used to compare demographics among groups. Statistical significance was defined as a $P$ value $<0.05$.

\section{Results}

Vestibular neuritis

The clinical characteristics of each group of VN patients are summarized in Table 1. There were 28, 12 and 24 patients in groups A, B and C, respectively. There were no significant differences in demographics among the groups (age, $P=0.613$; sex, $P=0.429)$. The mean UW of groups A-C 
was $69.2,71.3$ and $72.7 \%$, respectively $(P=0.895)$. The ratio of abnormal UW in groups A-C was 28/28 (100\%), $10 / 12(83.3 \%)$ and 19/24 (79.2\%), respectively. The trend to decreasing caloric abnormality was significant $(P=0.016)$. The mean IOD in groups $\mathrm{A}$ to $\mathrm{C}$ was 13.2 , 5.7 and $4.6^{\circ}$, respectively. The mean IOD differed significantly between group A and either group $\mathrm{B}(P=0.003)$ or $\mathrm{C}(P<0.001)$, but not between groups $\mathrm{B}$ and $\mathrm{C}(P=0.806)$. The OT abnormality decreased significantly according to the group $(P<0.001)$. Significant dissociation between OT and UW was observed among the groups (Table 1). There was a linear positive correlation between IOD and UW in group A $(r=0.749, P<0.001)$, but not in groups $\mathrm{B}(r=0.234$, $P=0.464)$ and C ( $r=0.304, P=0.149)($ Fig. 1$)$.

Ménière's disease

The clinical characteristics of each group of MD patients are summarized in Table 2. Of the 67 patients with MD, there were 16, 21 and 30 patients in groups A, B and C, respectively. There were no significant differences in demographics among the groups (age, $P=0.959$; sex, $P=0.579$ ). The mean $\mathrm{UW}$ in groups $\mathrm{A}$ to $\mathrm{C}$ was $28.4,23.0$ and $36.7 \%$, respectively $(P=0.315)$. The ratio of abnormal UW in groups A-C was 7/16 (43.8\%), 5/21 (23.8\%) and $16 / 30(53.3 \%)$, respectively $(P=0.334)$. The mean IOD in groups $\mathrm{A}-\mathrm{C}$ was $5.5,5.6$ and $3.7^{\circ}$, respectively $(P=0.276)$. The ratio of abnormal OT in groups A-C was $3 / 16(18.8 \%)$, $4 / 21(19.0 \%)$ and 5/30 (16.7\%), respectively $(P=0.841)$. The dissociation between OT and UW was not significant among the groups $(P=0.114)$ (Table 1$)$. A linear positive correlation between IOD and UW was found in group $\mathrm{C}(r=0.511, P=0.004)$, but not in groups A $(r=0.413$, $P=0.112)$ and $\mathrm{B}(r=0.118, P=0.610)$ (Fig. 2$)$.

\section{Discussion}

This study examined the correlation between static imbalance and dynamic disturbance according to the time course
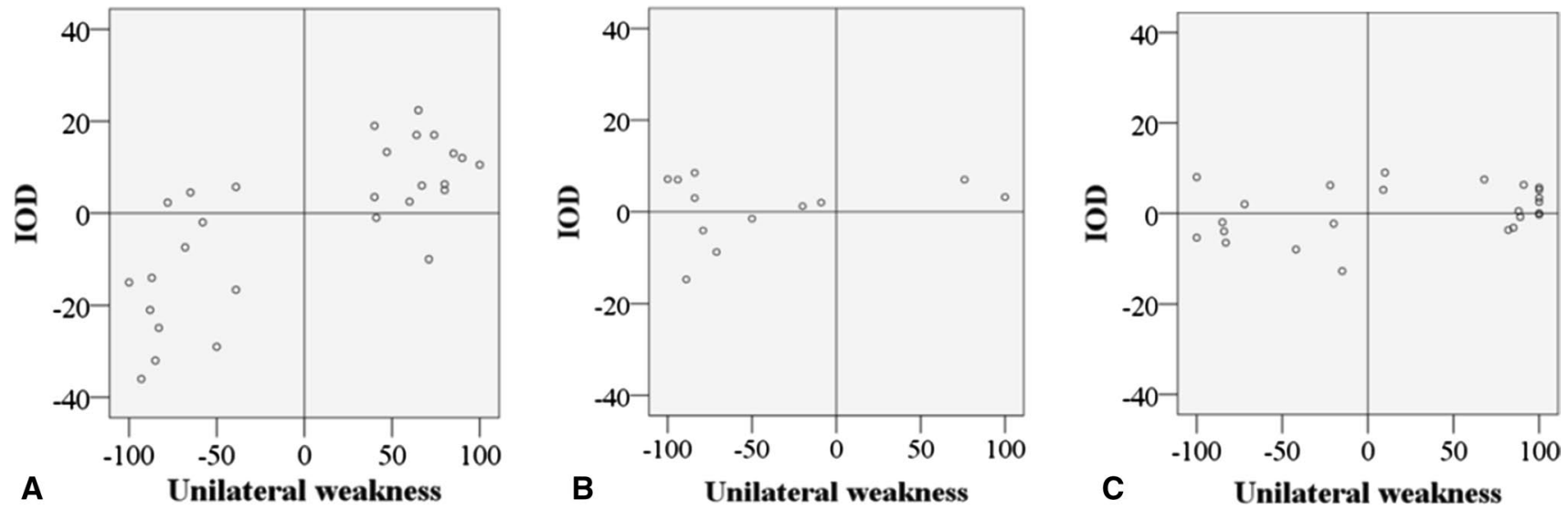

Fig. 1 Interocular difference (IOD) and caloric unilateral weakness (UW) according to the time course in vestibular neuritis. A linear correlation between IOD and UW is observed in group A (within

7 days of vertigo onset) $(r=0.749, P<0.001)(\mathbf{a})$, but not in group B (8-30 days) $(r=0.234, P=0.464)$ (b) or $\mathrm{C}$ (over 30 days) $(r=0.304, P=0.149)(\mathbf{c})$
Table 2 Demographics, caloric unilateral weakness and ocular torsion in patients with Ménière's disease

$M D$ Ménière's disease, $S D$ standard deviation, $I O D$ interocular difference

\begin{tabular}{lllll}
\hline & $\begin{array}{l}\text { Group A } \\
(n=16)\end{array}$ & $\begin{array}{l}\text { Group B } \\
(n=21)\end{array}$ & $\begin{array}{l}\text { Group C } \\
(n=30)\end{array}$ & $P$ value \\
\hline Age, mean \pm SD (year) & $54.1 \pm 12.3$ & $54.3 \pm 13.2$ & $55.1 \pm 12.6$ & 0.959 \\
Sex (M:F) & $9: 7$ & $17: 4$ & $16: 14$ & 0.579 \\
Unilateral weakness & & & $36.7 \pm 34.3$ & 0.315 \\
Mean \pm SD $(\%)$ & $28.4 \pm 31.8$ & $23.0 \pm 28.0$ & $16 / 30(53.3)$ & 0.334 \\
Abnormal & $7 / 16(43.8)$ & $5 / 21(23.8)$ & & \\
Ocular torsion & & & $3.7 \pm 3.9$ & 0.276 \\
Mean IOD $\left(^{\circ}\right)$ & $5.5 \pm 6.0$ & $5.6 \pm 4.5$ & $5 / 30(16.7)$ & 0.841 \\
Abnormal & $3 / 16(18.8)$ & $4 / 21(19.0)$ & $15 / 30(50.0)$ & 0.114 \\
Dissociation $(\%)$ & $4 / 16(25.0)$ & $9 / 21(42.9)$ &
\end{tabular}



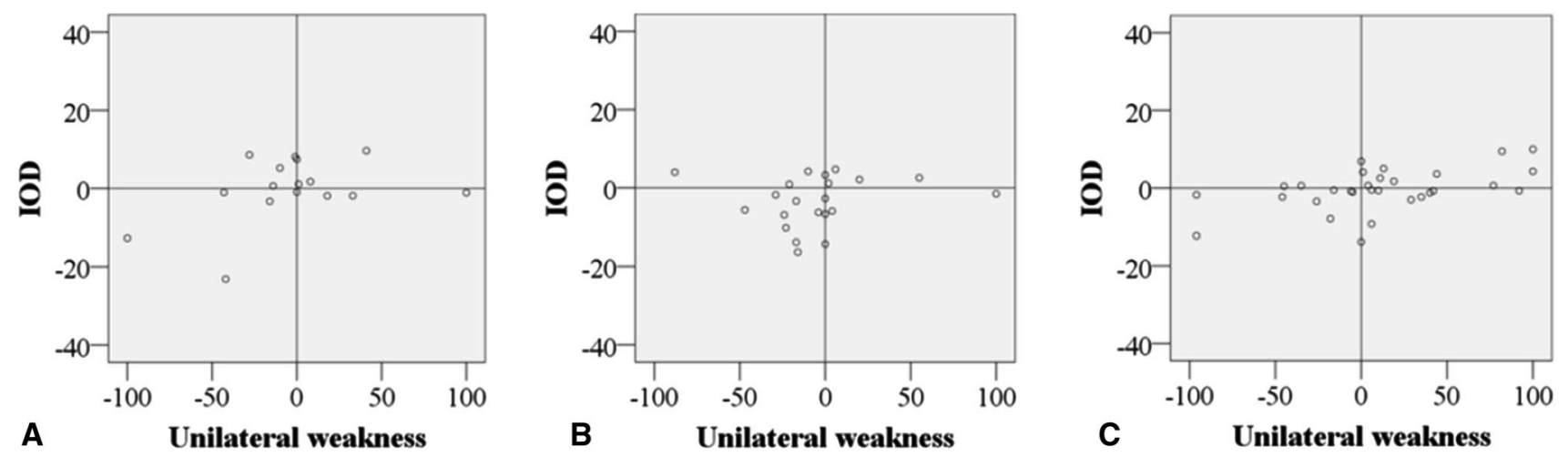

Fig. 2 Interocular difference (IOD) and caloric unilateral weakness (UW) according to the time course in Ménière's disease. A linear correlation between IOD and UW is observed in group C (over 30 days)

of two common peripheral vestibular disorders: VN, characterized by acute prolonged vestibulopathy; and MD, representing recurrent fluctuating vestibulopathy. In our patients, OT determined by fundus photography recovered more rapidly than UW and it almost invariably resolved over time in VN. This finding is in close agreement with those of the previous study $[2,4,11]$.

A linear correlation between the degree of OT and severity of UW was seen within 7 days of vertigo onset in VN. Over time, the association disappeared and dissociation between OT and UW was found because the central compensation restored the resting activity of the vestibular nucleus before the peripheral vestibular function improved.

In MD, fewer than half of the patients had an abnormal UW and the mean severity of UW of groups A, B and C was 28,23 and $37 \%$, respectively. The frequency of an abnormal UW in MD in our series was comparable with earlier reports that approximately half of the patients had unilateral vestibular hypofunction seen on bithermal caloric testing $[12,13]$. Furthermore, the differences among groups were not significant. The proportion of MD patients with an abnormal OT ranged from 16.7 to $19.0 \%$ and did not differ significantly among the groups. Therefore, OT did not differ regardless of recent vertigo attacks, and no central compensation accompanying peripheral vestibular loss was evident in MD. VN is a paresis of the vestibular nerve and the peripheral deafferentation in $\mathrm{VN}$ accompanies subsequent central compensation. Considering the pathophysiological mechanism of MD, which is thought to be endorgan dysfunction due to endolymphatic hydrops and the selective loss of type two hair cells $[14,15]$, the peripheral dysfunction does not seem to be severe.

Comparing the OT results between MD and VN in group $\mathrm{C}$, in which the latest true vertigo attack occurred more than 30 days earlier, the static imbalance shown by OT was higher in MD patients $(5 / 30,16.7 \%)$ than in VN patients $(r=0.511, P=0.004)(\mathbf{c})$, but not in group A (within 7 days of vertigo onset) $(r=0.413, P=0.112)$ (a) or B (8 to 30 days) $(r=0.118$, $P=0.610)(\mathbf{b})$

$(2 / 24,8.3 \%)$. Although this difference did not reach statistical significance, it suggests that recurrent subclinical spells, which depend on fluctuations in the endolymphatic pressure, inhibit central compensation and reduce vestibular function progressively in MD patients.

$\mathrm{VN}$ can recur on the same side, albeit rarely [16, 17]. Nevertheless, if vestibular function recovers fully after $\mathrm{VN}$, the same pattern of dissociation between OT and UW can be expected when VN recurs in the same ear. In MD, severities of vertigo attacks may be variably accompanied. The abnormality in OT was only $19 \%$ even in acute phase of true vertigo attack and it was not correlated with UW on caloric test. The frequency of adjuvant spells is usually higher than that of true vertigo attacks in most patients. In adjuvant spells, spontaneous nystagmus is usually nil or absent and OT would be within normal range. Considering vestibular migraine or migraine equivalent is frequently associated with MD, recurrent mild vertigo attacks in MD without abnormality in OT and UW can be explained by the association of migraine episodes. Therefore, the severity of the initial OT and the chronological dissociation pattern can be a good indicator to differentiate acute vertiginous attack of VN from that of MD.

\section{Conclusion}

In VN patients, OT to the lesion side was a prominent finding and correlated with the severity of the unilateral vestibular weakness on caloric testing during the acute phase, while OT abnormality decreased over time and the dissociation between OT and UW abnormalities became more prominent with central compensation. Unlike VN, no OT abnormality was consistently observed in acute MD patients experiencing a recent vertigo attack, and the OT and UW abnormalities were not correlated in the acute 
phase of MD. This different pattern in the OT abnormality is an additional useful finding for differentiating the ongoing pathogenic mechanism in patients experiencing recent, severe vertigo attacks. That is, the OT abnormality is remarkable during the acute phase of $\mathrm{VN}$, whereas it might not be remarkable immediately after a vertigo attack in a fluctuating vestibulopathy such as MD.

Conflict of interest On behalf of all authors, the corresponding author states that there are no conflicts of interest.

\section{References}

1. Sharpe JA, Kumar S, Sundaram AN (2011) Ocular torsion and vertical misalignment. Curr Opin Neurol 24(1):18-24. doi:10.109 7/WCO.0b013e328341e2b2

2. Kim HA, Hong JH, Lee H, Yi HA, Lee SR, Lee SY, Jang BC, Ahn BH, Baloh RW (2008) Otolith dysfunction in vestibular neuritis: recovery pattern and a predictor of symptom recovery. Neurology 70(6):449-453. doi:10.1212/01.wnl.0000297554.21221.a0

3. Curthoys IS, Dai MJ, Halmagyi GM (1991) Human ocular torsional position before and after unilateral vestibular neurectomy. Exp Brain Res 85(1):218-225

4. Choi KD, Oh SY, Kim HJ, Koo JW, Cho BM, Kim JS (2007) Recovery of vestibular imbalances after vestibular neuritis. Laryngoscope 117(7):1307-1312. doi:10.1097/MLG.0b013e318 05c08ac

5. Murofushi T, Iwasaki S, Ushio M (2006) Recovery of vestibular evoked myogenic potentials after a vertigo attack due to vestibular neuritis. Acta Otolaryngol 126(4):364-367. doi:10.1080/00016480500417189
6. Tahara T, Sekitani T, Imate Y, Kanesada K, Okami M (1993) Vestibular neuronitis in children. Acta Otolaryngol Suppl 503:49-52

7. Semaan MT, Megerian CA (2011) Meniere's disease: a challenging and relentless disorder. Otolaryngol Clin N Am 44(2):383403, ix. doi:10.1016/j.otc.2011.01.010

8. Strupp M, Brandt T (1999) Vestibular neuritis. Adv Otorhinolaryngol 55:111-136

9. Committee on Hearing and Equilibrium guidelines for the diagnosis and evaluation of therapy in Meniere's disease. American Academy of Otolaryngology-Head and Neck Foundation, Inc (1995). Otolaryngol Head Neck Surg 113 (3):181-185

10. Koo JW, Kim JS, Hong SK (2011) Vibration-induced nystagmus after acute peripheral vestibular loss: comparative study with other vestibule-ocular reflex tests in the yaw plane. Otol Neurotol 32(3):466-471. doi:10.1097/MAO.0b013e31820d9685

11. Dutia MB (2010) Mechanisms of vestibular compensation: recent advances. Curr Opin Otolaryngol Head Neck Surg 18(5):420 424. doi:10.1097/MOO.0b013e32833de71f

12. Sajjadi H, Paparella MM (2008) Meniere's disease. Lancet 372(9636):406-414. doi:10.1016/S0140-6736(08)61161-7

13. de Sousa LC, Piza MR, da Costa SS (2002) Diagnosis of Meniere's disease: routine and extended tests. Otolaryngol Clin N Am 35(3):547-564

14. Merchant SN (1999) A method for quantitative assessment of vestibular otopathology. Laryngoscope 109(10):1560-1569

15. Tsuji K, Velazquez-Villasenor L, Rauch SD, Glynn RJ, Wall C 3rd, Merchant SN (2000) Temporal bone studies of the human peripheral vestibular system: Meniere's disease. Ann Otol Rhinol Laryngol Suppl 181:26-31

16. Halmagyi GM, Weber KP, Curthoys IS (2010) Vestibular function after acute vestibular neuritis. Restor Neurol Neurosci 28(1):3746. doi:10.3233/RNN-2010-0533

17. Goddard JC, Fayad JN (2011) Vestibular neuritis. Otolaryngol Clin N Am 44(2):361-365, viii. doi:10.1016/j.otc.2011.01.007 